\title{
Circular RNA circRHOBTB3 is downregulated in hepatocellular carcinoma and suppresses cell proliferation by inhibiting miR-18a maturation
}

\author{
Gang $\mathrm{Hu}^{1+}$, Shusen $\mathrm{Zhai}^{2+}$, Sheng $\mathrm{Yu}^{3^{*}} \mathbb{D}^{\mathrm{D}}$, Zhen Huang ${ }^{1}$ and Ran Gao
}

\begin{abstract}
Background: Circular RNA circRHOBTB3 has been characterized as a tumor suppressor in gastric cancer, while its role in hepatocellular carcinoma (HCC) is unknown. This study was carried out to analyze the role of circRHOBTB3 in HCC.

Methods: In this study, circRHOBTB3, mature miR-18a, and miR-18a precursor in HCC and paired non-cancer tissues were detected by RT-qPCR. The role of circRHOBTB3 in the production of mature miR-18a was explored by transfecting circRHOBTB3 expression vector into HCC cells, followed by RT-qPCR to determine the expression of mature miR-18a and miR-18a precursor. The role of circRHOBTB3 and miR-18a in HCC cell proliferation was studied using CCK-8 assay.

Results: CircRHOBTB3 was under-expressed in HCC compared to normal tissues. In HCC cells, circRHOBTB3 overexpression decreased mature miR-18a level but not miR-18a precursor. Cell proliferation analysis showed that circRHOBTB3 overexpression decreased cell proliferation while miR-18a overexpression increased cell proliferation. Moreover, circRHOBTB3 suppressed the role of miR-18a in cell proliferation.

Conclusions: CircRHOBTB3 is downregulated in HCC and may suppress cell proliferation by reducing miR-18a production. Keywords: Hepatocellular carcinoma, circRHOBTB3, miR-18a, Precursor
\end{abstract}

\section{Introduction}

Hepatocellular carcinoma (HCC) is a major malignancy that originated from the liver and accounted for more than $85 \%$ of all liver cancer cases [1]. The incidence of HCC varies greatly worldwide, with the highest incidence rate observed in African countries and eastern Asia $[2,3]$, mainly owing to the high prevalence of

\footnotetext{
* Correspondence: ShengYuHepatobilio@163.com

${ }^{\dagger} \mathrm{Gang} \mathrm{Hu}$ and Shusen Zhai contributed equally to this work.

${ }^{3}$ Division of Hepatobiliopancreatic Surgery, Department of General Surgery, Nanfang Hospital, Southern Medical University, No. 1838 Guangzhou Avenue North, Guangzhou City, Guangdong Province 510515, People's Republic of China

Full list of author information is available at the end of the article
}

infections of hepatitis B virus (HBV) and hepatitis C virus $(\mathrm{HCV})$ in these regions [4]. Especially in China, $\mathrm{HCV}$ and HBV affect about 30 million and 93 million people, accounting for about $9 \%$ of the Chinese population. As a result, about 53\% HCC cases are believed to come from China $[5,6]$. Therefore, preventative and treatment approaches for HCC are urgently needed.

Although $\mathrm{HCV}$ and $\mathrm{HBV}$ infections are the main risk factors for HCC, they are not sufficient for its occurrence and development $[5,6]$. HCC is characterized by its hypervascularity and hepatocarcinogenesis. Receptor tyrosine kinases (RTKs) signaling pathways, such as Ras/ Raf/MEK/ERK, Jak/Stat, and PI3K/AKT/mTOR, could

C C The Author(s). 2021 Open Access This article is licensed under a Creative Commons Attribution 4.0 International License, which permits use, sharing, adaptation, distribution and reproduction in any medium or format, as long as you give appropriate credit to the original author(s) and the source, provide a link to the Creative Commons licence, and indicate if changes were made. The images or other third party material in this article are included in the article's Creative Commons licence, unless indicated otherwise in a credit line to the material. If material is not included in the article's Creative Commons licence and your intended use is not permitted by statutory regulation or exceeds the permitted use, you will need to obtain permission directly from the copyright holder. To view a copy of this licence, visit http://creativecommons.org/licenses/by/4.0/. The Creative Commons Public Domain Dedication waiver (http://creativecommons.org/publicdomain/zero/1.0/) applies to the data made available in this article, unless otherwise stated in a credit line to the data. 
activate many angiogenic signaling pathways, resulting in tumor cell proliferation, invasion, and metastasis [7, 8]. These data provide rational targets for innovative HCC therapies. mTOR inhibitors appear to be the most promising agents in this category [9-11]. Although many efforts have been made in molecularly targeted therapy in $\mathrm{HCC}$, the effective targets for $\mathrm{HCC}$ remain lacking. As covalently closed RNAs with no or limited protein-coding capacity, circular RNAs (circRNAs) are emerging novel targets for targeted cancer therapy, mainly owing to their roles in regulating gene expression $[12,13]$. In a recent study, Deng et al. reported a novel circRNA, circRHOBTB3 originated from exon 6 and exon 7 of RHOBTB3 gene as a tumor suppressor in gastric cancer [14]. Our preliminary microarray analysis revealed that circRHOBTB3 expression is altered in HCC (Fig.S1), and circRHOBTB3 is inversely correlated with miR-18a (data not shown).

miR-18a is one of the most conserved and multifunctional miRNAs in the polycistronic miR-17-92 cluster and is frequently overexpressed in malignant tumors [15]. Studies have shown that miR-18a is overexpressed in non-small-cell lung cancer (NSCLC) [16], cervical cancer (CC) [17], and gastric cancer (GC) [18]. Savitsky $D$ et al. have verified that the $3^{\prime}$-UTR of IRF2 is a target of miR-18a, which can promote NSCLC development via downregulating IRF2 [19]. Mezache $L$ et al. confirmed that miR-18a indirectly upregulates PD-L1 expression by activating the PI3K/AKT, MEK/ ERK, and $\mathrm{Wnt} / \beta$-catenin pathways, resulting in $\mathrm{CC}$ progression [20]. In addition, another study showed that miR-18a is an oncogene and plays a vital role in GC development by negatively regulating the protein inhibitor of activated STAT (PIAS)-3 and thereby modulating STAT3 target genes [18]. Liu L et al. showed that miR-18a is upregulated in human HCC tissues and cell lines and promotes HCC cell proliferation and migration by targeting KLF4 and its p21 [21]. Despite emerging evidence about the role of miRNAs in cancer progression, fewer studies have explored the functions of miR-18a than other miRNAs in the miR-17-92 cluster in cancer development [15]. In this study, we focused on analyzing the crosstalk between circRHOBTB3 and miR-18a in HCC.

\section{Materials and methods HCC patients}

A total of $62 \mathrm{HCC}$ patients (38 to 66 years; median age: 52 years), including 42 males and 20 females, at Nanfang Hospital, Southern Medical University from May 2018 to May 2020 were enrolled in the study. These patients included 28 cases at AJCC stage I or II and 34 cases at III or IV. HBV infection was observed in 22 cases, and $\mathrm{HCV}$ infection was observed in 30 cases. To exclude other factors that may affect the expression of target genes, patients with initiated therapy or other severe clinical disorders, such as diabetes, heart diseases, severe infections, and other malignancies were excluded. The study was approved by the Ethics Committee of Nanfang Hospital. All patients signed the informed consent.

\section{HCC tissue collections and HCC cell lines}

$\mathrm{HCC}$ and adjacent non-cancer tissues were collected by aspirating 3 times in different directions using fine needles from all patients prior to therapies. Tissue samples were confirmed histopathologically using hematoxylineosin (H\&E) staining and stored in liquid nitrogen prior to the subsequent assays.

SNU-449 and SNU-387 (ATCC) cell lines were used as HCC cell models in this study. Normal liver THLE-2 cell line (ATCC) was used as the control. Cells were cultured in RPMI- 1640 medium with $10 \% \mathrm{FBS}$ at $37^{\circ} \mathrm{C}$ and $5 \% \mathrm{CO}_{2}$.

\section{Lipofectamine 2000-mediated transfections}

CircRHOBTB3 expression vector was established with pcDNA3.1 (Addgene) as the backbone. MiR-18a mimic and negative control (NC) were purchased from SigmaAldrich. To overexpress circRHOBTB3 and miR-18a, SNU-449 and SNU-387 were transfected with either $1 \mu \mathrm{g}$ expression vector or $40 \mathrm{nM}$ miRNA using Lipofectamine 2000 (Invitrogen). Empty vector or NC miRNA transfected cells were used as the NC cells.

\section{RNA preparations}

RNAs were extracted from SNU-449 and SNU-387 cells and tissues collected from the $62 \mathrm{HCC}$ patients using Ribozol reagent (VWR Life Science) and treated with DNase I (Invitrogen) for $1 \mathrm{~h}$ at $37^{\circ} \mathrm{C}$ to completely remove genomic DNAs. RNA integrity was examined by electrophoresis on 6\% urea-PAGE gel, and RNA purity was reflected by OD260/280 ratio.

\section{qRT-PCR}

For mRNA detection, RNA samples were reverse transcribed (RTs) using the SSRT-III-RT system (Invitrogen) and subjected to qPCR analysis with LightCycler 480 SYBR Green I Master (Roche Life Science). The relative mRNA expression was determined using the $2^{-\Delta \Delta C t}$ method with GAPDH as the reference. The PCR primers were circRHOBTB3 forward 5'-GAAGTTGAAAGATT CTGGGGA-3' and reverse 5'-ACTGGCAGCAGAACAGCAAG-3' and GAPDH forward 5'-GTCTCCTCTG ACT TCAACAGCG-3' and reverse 5' -ACCACCCTGT TGCTGTAGCCAA-3'. The levels of miR-18a precursor and mature miR-18a were examined by qRT-PCRs using All-in-One ${ }^{\mathrm{Tm}}$ miRNA qRT-PCR Detection Kit (GeneCopoeia). The relative miRNA expression was determined using the $2^{-\Delta \Delta \mathrm{Ct}}$ method with U6 as the reference. The 
PCR primers were miR-18a precursor forward 5 '-TGTT CTAAGGTGCATCTAG-3' and reverse 5' ' $^{\prime}$ TGCCAG AAGGAGCACTTAG-3', miR-18a forward 5' -CACGCA TAAGG TGCATCTAGTGC-3' and reverse 5'-CCAG TGCAGGGTCCGAGGTA-3', and U6 forward 5'CTCGCTTCGGCAGCACA-3' and reverse 5' ${ }^{\prime}$-AACG CTTCACGAATTTG CGT-3'. All qPCRs were performed in three technical replicates.

\section{Cell proliferation assay}

At $48 \mathrm{~h}$ of post-transfection, cell proliferation was analyzed using CCK-8 kits (Sigma-Aldrich). In brief, 5000 cells were placed in each well of 96-well plates. Cell proliferation was measured every $24 \mathrm{~h}$ for 4 times at $2 \mathrm{~h}$ after the addition of $10 \%$ CCK- 8 by measuring the optical density (OD) at $450 \mathrm{~nm}$.

\section{Statistical analysis}

CircRHOBTB3 and miR-18a expression in tissues and cell lines fulfilled the normal distribution, which was analyzed by the Kolmogorov-Smirnov test in SPSS software. Thus, circRHOBTB3 and miR-18a expression in tissues from HCC patients $(n=62)$ were compared by paired $\mathrm{t}$ test. Comparisons between two independent groups were performed by unpaired $t$ test. ANOVA
Tukey's test was used to compare differences among multiple transfection groups. $P<0.05$ was statistically significant.

\section{Results}

HCC exhibited downregulated circRHOBTB3 expression CircRHOBTB3 expression in paired tissues from HCC patients $(n=62)$ was analyzed by RT-qPCR. CirCRHOBTB3 was significantly under-expressed in HCC tissues (Fig. 1A, $p<0.01$ ). CircRHOBTB3 expression in HCC tissues was not significantly different between AJCC stages (I or II vs. III or IV; Fig. $1 \mathrm{~B}, p<0.01$ ). Among the HCC patients, HBV infection was observed in 22 cases, $\mathrm{HCV}$ infection was observed in 30 cases, and non-infection was observed in 10 cases. No significant differences in circRHOBTB3 expression were found among these three groups (Fig. 1C). CircRHOBTB3 levels in SNU-448, SNU-387, and normal liver cell line THLE-2 were measured by RT-qPCR. The results showed that circRHOBTB3 was also downregulated in SNU-448 and SNU-387 cell lines compared to normal THLE-2 cell line (Fig. 1D). It is worth noting that RHOBTB3 mRNA was also downregulated in HCC cell lines (Fig. S2).
A
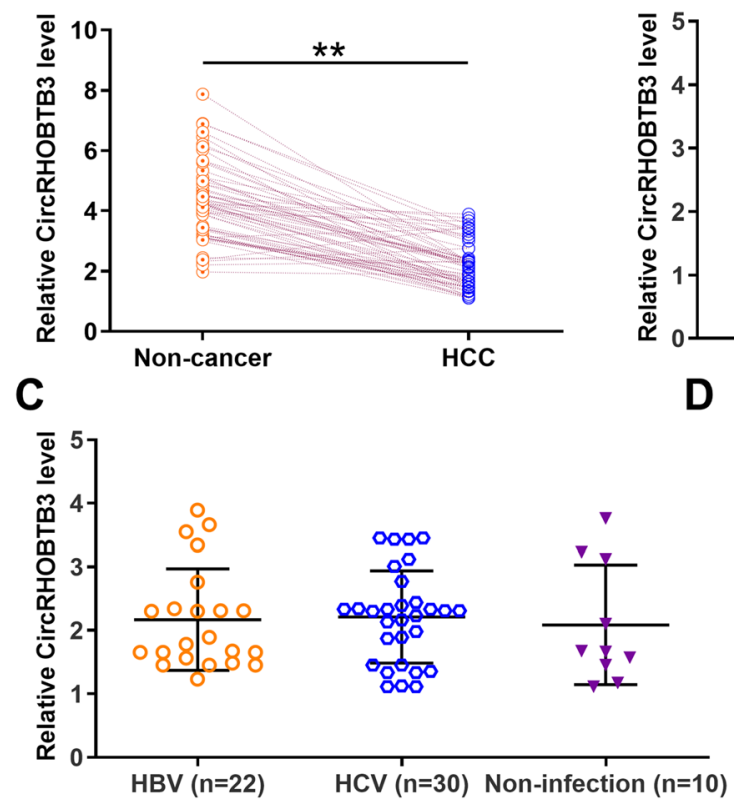

B
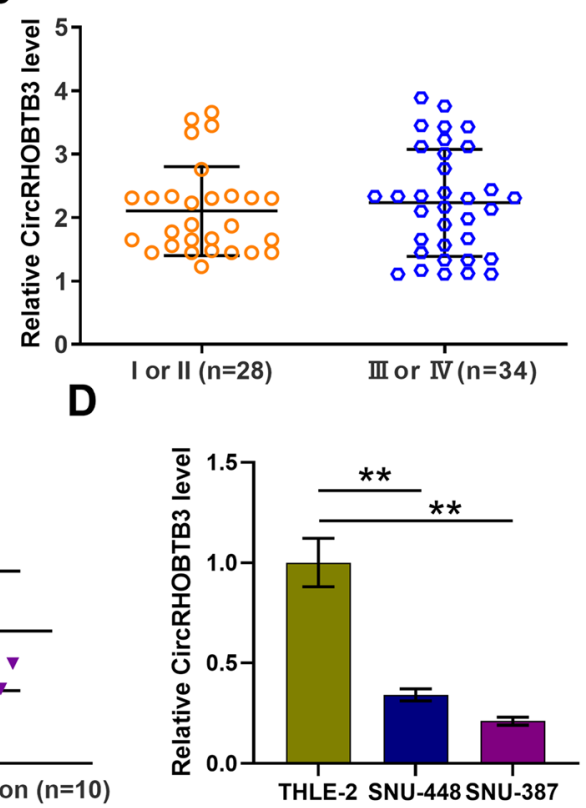

Fig. 1 CircRHOBTB3 expression was downregulated in HCC but was not affected by clinical stages and infections of HBV and HCV. CircRHOBTB3 expression in paired HCC and non-tumor tissues from HCC patients $(n=62)$ was analyzed by qRT-PCR $(\mathbf{A})$. CircRHOBTB3 expressions at AJCC stage I or II and stage III or IV HCC tissues were compared (B). CircRHOBTB3 expressions were compared in HCC tissues from patients with HCV infection, HBV infection, and non-infection (C). CircRHOBTB3 levels in SNU-448, SNU-387, and normal liver cell line THLE-2 were measured by qRTPCR (D). CircRHOBTB3 levels were expressed as the average of three technical qRT-PCR replicates in tissue samples from HCC patients $(n=62)$ or in cell lines. GAPDH was used for normalization. ${ }^{* *} p<0.01$ 
Both mature miR-18a and miR-18a precursor were upregulated in HCC, but circRHOBTB3 was only inversely correlated with mature miR-18a

Mature miR-18a and miR-18a precursor expressions in paired tissues from HCC patients $(n=62)$ were also analyzed by RT-qPCRs. Mature miR-18a (Fig. 2A) and miR18a precursor (Fig. 2B) were significantly overexpressed in HCC tissues than in non-tumor tissues $(p<0.01)$. Correlation analysis performed by Pearson's correlation coefficient showed thatcircRHOBTB3 was inversely correlated with mature miR-18a (Fig. 2C), but not miR-18a precursor (Fig. 2D) across HCC tissues. Therefore, we hypothesized that circRHOBTB3 is likely involved in miR-18a maturation.

\section{CircRHOBTB3 overexpression decreased the production of} mature miR-18a

To study the role of circRHOBTB3 in regulating miR18a maturation, SNU-449 and SNU-387 cells were transfected with circRHOBTB3 expression vector or miR-18a mimic, followed by RT-qPCRs to confirm the overexpression of circRHOBTB3 and mature miR-18a (Fig. 3A, $p<0.05)$. CircRHOBTB3 overexpression significantly decreased the level of mature miR-18a (Fig. 3B, $p<0.05$ ), but not miR-18a precursor (Fig. 3C). Therefore, circRHOBTB3 may suppress miR-18a maturation in HCC cells. In addition, circRHOBTB3 had no effect on the levels of mature miR-18a and miR-18a precursor in THLE-2 cell lines (Fig. S2).

\section{CircRHOBTB3 overexpression decreased HCC cell proliferation}

The role of circRHOBTB3 and miR-18a in the proliferation of SNU-449 and SNU-387 cells was analyzed by CCK-8 assay. CircRHOBTB3 overexpression decreased cell proliferation, while miR-18a overexpression increased cell proliferation. Moreover, circRHOBTB3 suppressed the role of miR-18a in cell proliferation (Fig. 4, $p<0.05)$. However, CircRHOBTB3 and miR-18a overexpression have no effect on the proliferation of THLE-2 cell lines (Fig. S2).

\section{Discussion}

We analyzed the differential expression of circRHOBTB3 in HCC and studied its crosstalk with miR18a. The results showed that circRHOBTB3 was underexpressed in HCC and inhibited HCC cell proliferation by suppressing miR-18a maturation.
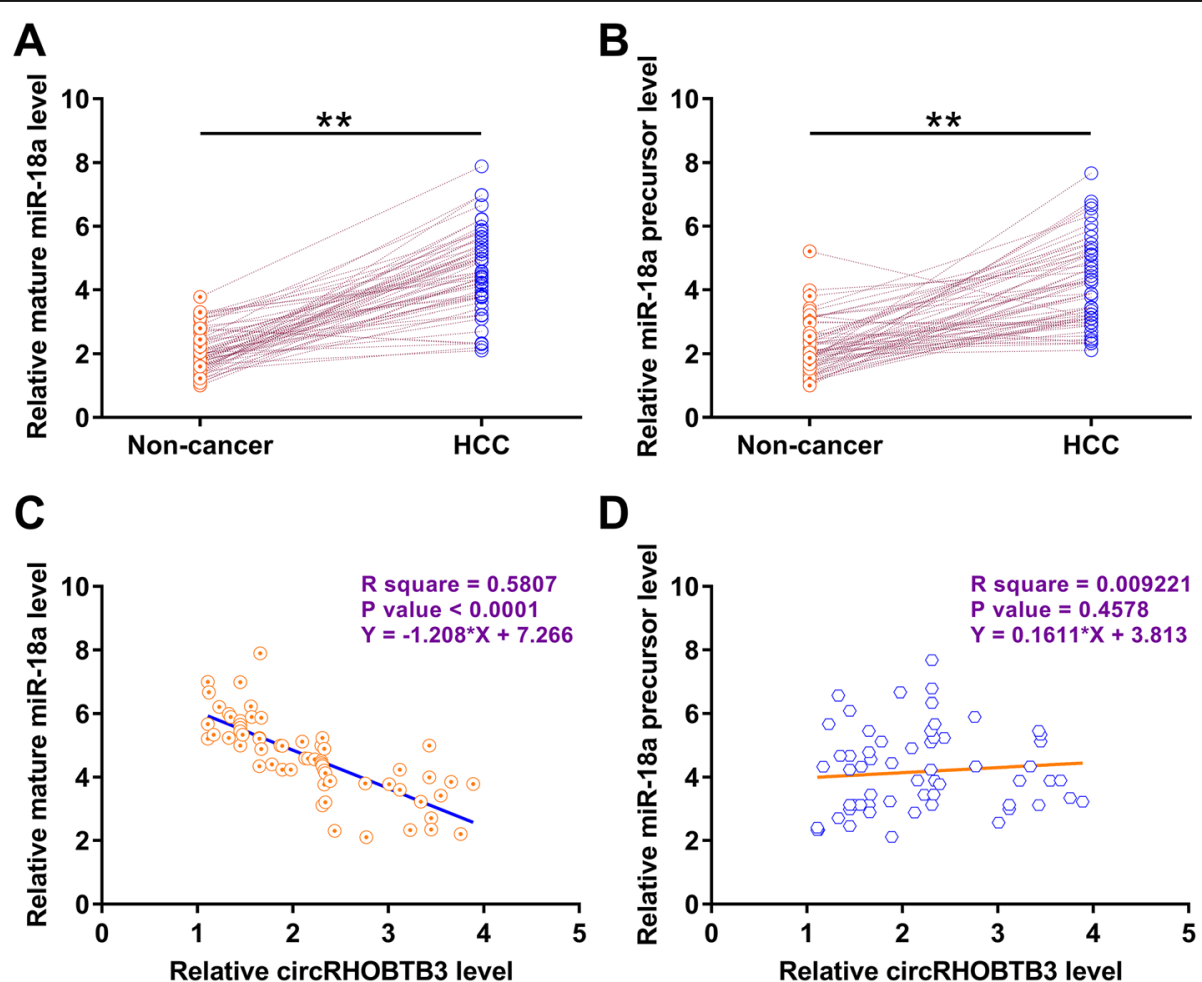

Fig. 2 Mature miR-18a and miR-18a precursor were both upregulated in HCC, but circRHOBTB3 was only inversely correlated with mature miR18a. Mature miR-18a (A) and miR-18a precursor (B) expressions in paired tissues from HCC patients $(n=62)$ were analyzed by qRT-PCRs. Expression levels of mature miR-18a and miR-18a precursor in tissue samples from HCC patients $(n=62)$ were expressed as the average of three technical qRT-PCR replicates. U6 was used for normalization. ${ }^{* *} p<0.01$. Correlations between circRHOBTB3 and mature miR-18a (C) or miR-18a precursor (D) were analyzed by Pearson's correlation coefficient 

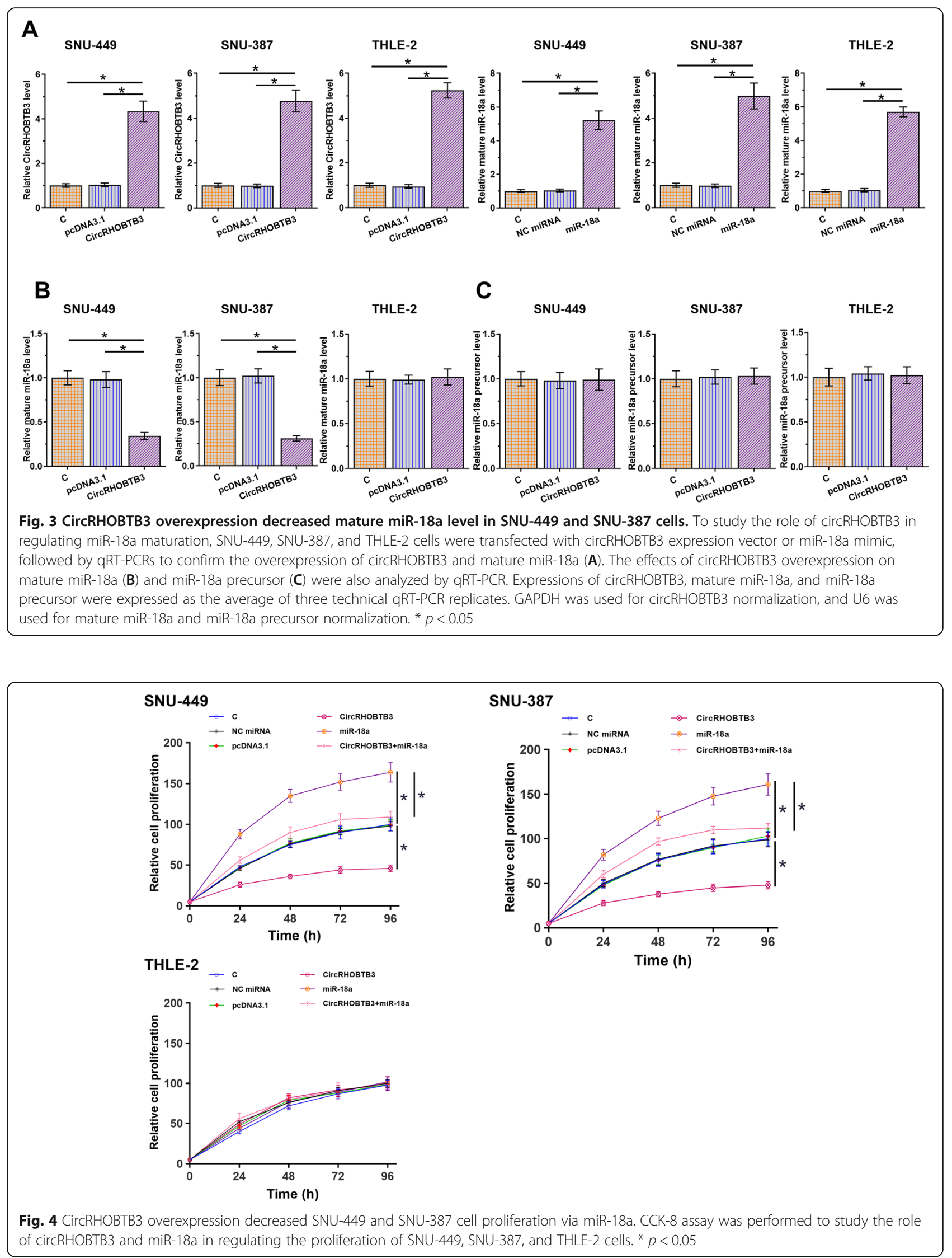
CircRHOBTB3 is significantly downregulated in gastric cancer [14]. In addition, circRHOBTB3 overexpression sponges miR-654-3p to suppress the growth of gastric tumor, suggesting the role of circRHOBTB3 as a tumor suppressor in this malignancy [14]. This study, for the first time, reported circRHOBTB3 downregulation in HCC. Interestingly, circRHOBTB3 expression was not significantly affected by clinical stags, suggesting that circRHOBTB3 may participate in the whole process of HCC development but not specific stages. Moreover, circRHOBTB3 expression was also not significantly affected by HBV and HCV infections, the major risk factors for HCC $[5,6]$. Therefore, circRHOBTB3 may participate in HCC through $\mathrm{HBV}$ - and HCVindependent pathways.

MiR-18a has been characterized as an oncogenic miRNA in many cancers, including HCC [21]. MiR-18a is overexpressed in HCC and targets KLF4 to promote tumor metastasis and growth in HCC [21]. Consistently, our study confirmed the upregulation of both miR-18a precursor and mature miR-18a in HCC. Therefore, the miR-18a may be upregulated at the transcription level from the process from pri-miRNA to miRNA precursor.

Besides regulating gene expression at transcriptional and translational levels, circRNAs may also sponge miRNAs to participate in human cancers, including HCC [22]. For instance, circRNA circMTO1 may sponge miR9 to inhibit the progression of HCC [22]. In this study, we reported the interaction between circRHOBTB3 and miR-18a. Interestingly, circRHOBTB3 is unlikely a sponge of miR-18a. Memczak $\mathrm{S}$ et al. found that CDR1as can function as a miR-7 sponge to reduce midbrain sizes in zebrafish, and this biological activity of CDR1as could be partially rescued by injecting miR-7 precursor [23]. Our results showed that circRHOBTB3 overexpression decreased mature miR-18a level, leading to reduced HCC cell proliferation while had no effect on miR-18a precursor level. These results suggested that the effect of circRHOBTB3 overexpression on HCC cell proliferation cannot be rescued by the miR-18a precursor. However, our results showed that the effect of circRHOBTB3 on $\mathrm{HCC}$ cell proliferation could be rescued by mature miR18a overexpression. Therefore, we believe that circRHOBTB3 overexpression-reduced HCC cell proliferation may be due to its role to suppress miR-18a maturation rather than its role as a molecular sponge of miR-18. Our findings revealed another type of interaction between circRHOBTB3 and miR-18a and suggested that circRHOBTB3 overexpression hinders the formation of mature miR-18a instead of miR-18a precursor, thereby reducing HCC cell proliferation. Because the formation of mature miRNAs requires transportation of miRNA precursors from the nucleus to the cytoplasm, we speculate that circRHOBTB3 may be involved in forming mature miRNAs from miRNA precursors. Our future studies will explore this possibility. Interestingly, we did not observe the increased level of miR-18a precursor along with decreased mature miR-18a in cells with circRHOBTB3 overexpression. It possibly indicated that circRHOBTB3 could also downregulate total miR18a. In addition, we found that RHOBTB3 mRNA level was downregulated in HCC line cells. MiRNAs regulate physiological and pathological processes via inhibiting target mRNA translation or promoting mRNA degradation [24]. In this study, we only revealed the interaction between circRHOBTB3 and miR-18a. The underlying mechanism for this interaction needs to be further explored.

\section{Conclusion}

In conclusion, circRHOBTB3 is under-expressed in HCC. Moreover, circRHOBTB3 may suppress the maturation of miR-18a to promote HCC cell proliferation.

\section{Supplementary Information}

The online version contains supplementary material available at https://doi. org/10.1186/s13027-021-00384-1.

Additional file 1 Fig. S1 The microarray results of circRHOBTB3 and miR-18a

Additional file 2 Fig. S2 RHOBTB3 mRNA expression in cell lines.

\section{Acknowledgments}

Not Applicable.

\section{Authors' contributions}

Gang Hu, Shusen Zhai, Ran Gao: study concepts, literature research, clinical studies, data analysis, experimental studies, manuscript writing and review; Sheng Yu: study design, literature research, experimental studies and manuscript editing; Zhen Huang: definition of intellectual content, clinical studies, data acquisition and statistical analysis. The author(s) read and approved the final manuscript.

Funding

Not Applicable.

Availability of data and materials

The data that support the findings of this study are available on request from the corresponding author: Sheng Yu, Division of Hepatobiliopancreatic Surgery, Department of General Surgery, Nanfang Hospital, Southern Medical University, No. 1838 Guangzhou Avenue North, Guangzhou City, Guangdong Province, 510,515, P. R. China. The data are not publicly available due to their containing information that could compromise the privacy of research participants.

\section{Declarations}

Ethical approval and consent to participate

The study was approved by the Ethics Committee of Nanfang Hospital, Southern Medical University. Informed consent was obtained from all individual participants included in this study. Procedures operated in this research were completed in keeping with the standards set out in the Announcement of Helsinki and the Laboratory Guidelines of Research in China.

Consent for publication

Not applicable. 


\section{Competing interests}

All authors have no conflicts of interest. We declare that we do not have any commercial or associative interest that represents a conflict of interest in connection with the work submitted.

\section{Author details}

'Department of General Surgery, Strategic Support Force Characteristic Medical Center, Beijing 100101, People's Republic of China. ${ }^{2}$ Department of Oncology, Strategic Support Force Characteristic Medical Center, Beijing 100101, People's Republic of China. ${ }^{3}$ Division of Hepatobiliopancreatic Surgery, Department of General Surgery, Nanfang Hospital, Southern Medical University, No. 1838 Guangzhou Avenue North, Guangzhou City, Guangdong Province 510515, People's Republic of China.

Received: 8 January 2021 Accepted: 7 June 2021

Published online: 29 June 2021

\section{References}

1. Balogh J, Victor D 3rd, Asham EH, Burroughs SG, Boktour M, Saharia A, et al. Hepatocellular carcinoma: a review. J Hepatocellular Carcinoma. 2016;3:4153. https://doi.org/10.2147/JHC.S61146.

2. Ozakyol A. Global epidemiology of hepatocellular carcinoma (HCC epidemiology). Journal of gastrointestinal cancer. 2017:48(3):238-40. https:// doi.org/10.1007/s12029-017-9959-0.

3. Yang JD, Roberts LR. Hepatocellular carcinoma: A global view. Nat Rev Gastroenterol Hepatol. 2010;7(8):448-58. https://doi.org/10.1038/nrgastro.201 0.100

4. Zampino R, Pisaturo MA, Cirillo G, Marrone A, Macera M, Rinaldi L, et al. Hepatocellular carcinoma in chronic HBV-HCV co-infection is correlated to fibrosis and disease duration. Ann Hepatol. 2015;14(1):75-82. https://doi. org/10.1016/S1665-2681(19)30803-8

5. Tanaka M, Katayama F, Kato H, Tanaka H, Wang J, Qiao YL, et al. Hepatitis B and $C$ virus infection and hepatocellular carcinoma in China: a review of epidemiology and control measures. Journal of epidemiology. 2011;21(6): 401-16. https://doi.org/10.2188/jea.JE20100190.

6. Wang FS, Fan JG, Zhang Z, Gao B, Wang HY. The global burden of liver disease: the major impact of China. Hepatology (Baltimore, Md). 2014;60(6): 2099-108.

7. Brechot C, Kremsdorf D, Soussan P, Pineau P, Dejean A, Paterlini-Brechot $P$, et al. Hepatitis B virus (HBV)-related hepatocellular carcinoma (HCC): molecular mechanisms and novel paradigms. Pathologie-biologie. 2010; 58(4):278-87. https://doi.org/10.1016/j.patbio.2010.05.001.

8. Rongrui L, Na H, Zongfang L, Fanpu J, Shiwen J. Epigenetic mechanism involved in the HBV/HCV-related hepatocellular carcinoma tumorigenesis. Curr Pharm Des. 2014;20(11):1715-25. https://doi.org/10.2174/138161281131 99990533.

9. Chan SL, Yeo W. Targeted therapy of hepatocellular carcinoma: present and future. J Gastroenterol Hepatol. 2012;27(5):862-72. https://doi.org/10.1111/ j.1440-1746.2012.07096.x.

10. Huynh H. Molecularly targeted therapy in hepatocellular carcinoma. Biochem Pharmacol. 2010;80(5):550-60. https://doi.org/10.1016/j.bcp.2010. 03.034.

11. Zhou Q, Lui WW, Yeo W. Targeting the PI3K/Akt/mTOR pathway in hepatocellular carcinoma. Future Oncol (London, England). 2011;7(10):1149_ 67.

12. He J, Xie Q, Xu H, Li J, Li Y. Circular RNAs and cancer. Cancer Lett. 2017;396: 138-44. https://doi.org/10.1016/j.canlet.2017.03.027.

13. Patop IL, Kadener S. circRNAs in Cancer. Curr Opin Genet Dev. 2018;48:1217. https://doi.org/10.1016/j.gde.2017.11.007.

14. Deng G, Mou T, He J, Chen D, Lv D, Liu H, et al. Circular RNA circRHOBTB3 acts as a sponge for miR-654-3p inhibiting gastric cancer growth. Journal of experimental \& clinical cancer research: CR. 2020;39(1):1. https://doi.org/1 0.1186/s13046-019-1487-2.

15. Shen $K$, Cao Z, Zhu R, You L, Zhang $T$. The dual functional role of MicroRNA-18a (miR-18a) in cancer development. Clinical and translational medicine. 2019;8(1):32. https://doi.org/10.1186/s40169-019-0250-9.

16. Liang C, Zhang X, Wang HM, Liu XM, Zhang XJ, Zheng B, et al. MicroRNA18a-5p functions as an oncogene by directly targeting IRF2 in lung cancer. Cell Death Dis. 2017;8(5):e2764. https://doi.org/10.1038/cddis.2017.145.

17. Dong $\mathrm{P}$, Xiong $Y, Y u$ J, Chen L, Tao T, Yi S, et al. Control of PD-L1 expression by miR-140/142/340/383 and oncogenic activation of the OCT4-miR-18a pathway in cervical cancer. Oncogene. 2018;37(39):5257-68. https://doi. org/10.1038/s41388-018-0347-4.

18. Wu W, Takanashi M, Borjigin N, Ohno SI, Fujita K, Hoshino S, et al. MicroRNA-18a modulates STAT3 activity through negative regulation of PIAS3 during gastric adenocarcinogenesis. Br J Cancer. 2013;108(3):653-61. https://doi.org/10.1038/bjc.2012.587.

19. Savitsky D, Tamura T, Yanai H, Taniguchi T. Regulation of immunity and oncogenesis by the IRF transcription factor family. Cancer Immunol Immunother. 2010;59(4):489-510. https://doi.org/10.1007/s00262-009-0804-6.

20. Mezache L, Paniccia B, Nyinawabera A, Nuovo GJ. Enhanced expression of PD L1 in cervical intraepithelial neoplasia and cervical cancers. Modern pathology : an official journal of the United States and Canadian Academy of Pathology, Inc. 2015;28(12):1594-602. https://doi.org/10.1038/modpa thol.2015.108

21. Liu L, Cai X, Liu E, Tian X, Tian C. MicroRNA-18a promotes proliferation and metastasis in hepatocellular carcinoma via targeting KLF4. Oncotarget. 2017; 8(40):68263-9. https://doi.org/10.18632/oncotarget.19293.

22. Han D, Li J, Wang H, Su X, Hou J, Gu Y, et al. Circular RNA circMTO1 acts as the sponge of microRNA-9 to suppress hepatocellular carcinoma progression. Hepatology (Baltimore, Md). 2017;66(4):1151-64.

23. Memczak S, Jens M, Elefsinioti A, Torti F, Krueger J, Rybak A, et al. Circular RNAs are a large class of animal RNAs with regulatory potency. Nature. 2013;495(7441):333-8. https://doi.org/10.1038/nature11928.

24. Di Leva G, Garofalo M, Croce CM. MicroRNAs in cancer. Annu Rev Pathol. 2014;9(1):287-314. https://doi.org/10.1146/annurev-pathol-012513-104715.

\section{Publisher's Note}

Springer Nature remains neutral with regard to jurisdictional claims in published maps and institutional affiliations.
Ready to submit your research? Choose BMC and benefit from:

- fast, convenient online submission

- thorough peer review by experienced researchers in your field

- rapid publication on acceptance

- support for research data, including large and complex data types

- gold Open Access which fosters wider collaboration and increased citations

- maximum visibility for your research: over $100 \mathrm{M}$ website views per year

At $\mathrm{BMC}$, research is always in progress.

Learn more biomedcentral.com/submissions 